\title{
Preface
}

\section{PET-Computed Tomography and PET-MR Imaging and Their Applications in the Twenty-First Century}

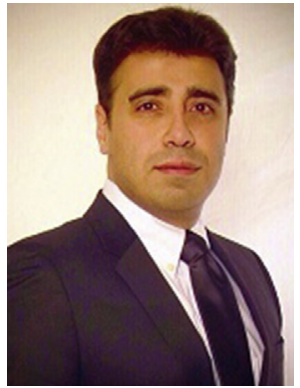

Ali Gholamrezanezhad, MD

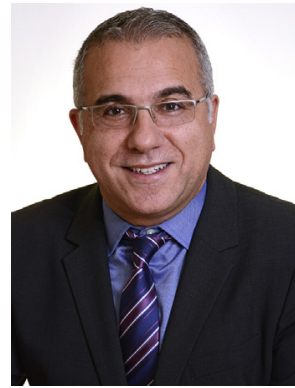

Ali Guermazi, MD, PhD

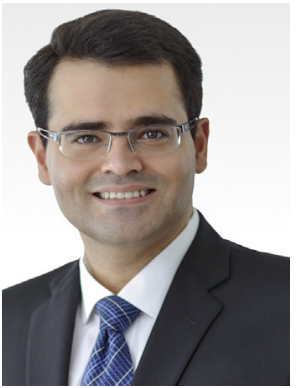

Ali Salavati, MD, MPH

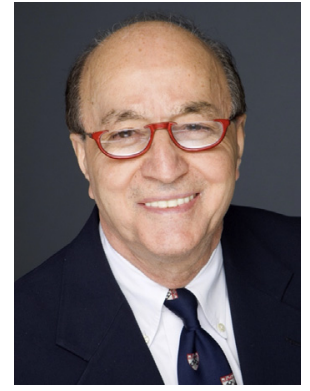

Abass Alavi, MD, MD (Hon), PhD (Hon), DSc (Hon)

Editors

Since the discovery of $x$-ray by Roentgen in 1895 , planar radiography has remained a major imaging technique in assessing skeletal abnormalities with reasonable success. However, poor contrast between diseased sites and the background results in low sensitivity of this modality in detecting early disease and monitoring its course over time. The introduction of computed tomography (CT) in 1971 by Hounsfield further enhanced the role of $\mathrm{x}$-ray-based disease assessment, and as such, XCT has played an important role in the day-to-day management of musculoskeletal (MSK) disorders. Since the early 1980s, when the first MR imaging instruments were introduced for human studies, the impact of imaging for examining soft tissue abnormalities in MSK disorders has been substantially enhanced. Currently, XCT and MR imaging are the main imaging modalities available in this domain but suffer from many deficiencies that need to be addressed by employing more advanced approaches. Since the early 1970s, 99m-Technitium (Tc)-labeled phosphates have been extensively used to detect benign and malignant disorders of the skeletal system. These radiotracers allow planar and tomographic imaging (SPECT [single-photon emission computed tomography]), but the quality of images generated is somewhat suboptimal for detection of the affected sites and quantification of the disease activity.

Fluorine18-sodium fluoride (18F-NaF), a positron-emitting tracer, was initially used in the early 1960s as a bone-seeking tracer but was abandoned soon after the introduction of $99 \mathrm{~m}$ Tc-labeled phosphates because of the limited availability of this compound and appropriate instruments. However, over the past four decades, with the introduction of PET imaging as a viable and successful molecular diagnostic modality, there is a resurgence of interest in using novel tracers in many domains, including the MSK system. This has resulted in the reintroduction of $18 \mathrm{~F}-\mathrm{NaF}$ for examining osseous abnormalities at the molecular level throughout the body. Furthermore, the introduction of $18 \mathrm{~F}$-fluorodeoxyglucose (FDG) in 1976 by the University of Pennsylvania investigators has allowed detection and monitoring of the course of a multitude of diseases and disorders, including those of soft tissue organs and the MSK system. Despite heavy emphasis being placed on FDG-PET for detection and characterization of various malignancies, increasingly, FDG is used for detection of infection and inflammation in many organs. The introduction of PET/CT in 
2001 and PET/MR imaging in the past decade, as two advanced imaging modalities that combine both molecular and structural aspects, has substantially enhanced the role of PET-based technology and is expected to revolutionize the disciplines of Orthopedics and Rheumatology.

Following the experience that has been gained by the introduction of these new technologies to medical imaging, it has become apparent that structural imaging alone is insensitive and nonspecific for many clinical applications, and as such, leads to undertreatment or overtreatment of many human maladies. By now, it has become apparent that $P E T$ is substantially more sensitive and specific than either CT or MR imaging in many organ disease entities, and therefore, it will play a major role in the future practice of medicine. Combining structural imaging with $\mathrm{PET}$ allows precise localization of molecular abnormalities and also quantification of disease activity. Accurate quantification provided by PET is another major asset of this modality and has become an essential element for measuring response to therapeutic interventions.

Originally, FDG was introduced as a molecular probe to examine central nervous system dysfunction due to Alzheimer disease, head injury, seizure disorders, and miscellaneous neuropsychiatric diseases. Since the 1980s, when whole body imaging with PET became feasible, FDG-PET has been primarily used to manage a multitude of malignant disorders and has truly revolutionized the discipline of medical, radiation, and surgical oncology over the years. Currently, the main indication for performing FDG-PET imaging deals with diagnosis, staging, assessment of response, and detection of recurrence in most suspected or proven malignancies. However, in the past two decades, it has become quite apparent that FDG is the tracer of choice for detecting inflammation due to infection or other underlying pathologic processes. Unfortunately, because of the limited experience and published data in this domain, no medical insurance coverage reimbursement exists for adopting FDG-PET imaging in the day-to-day practice of medicine. Therefore, there is a dire need to validate and provide convincing evidence for its validity and routine use in in the future.

Recently, $18 \mathrm{~F}-\mathrm{NaF}$ was approved as an acceptable procedure for detecting metastasis to the skeletal system, and it provides the main clinical indication for its use in medicine. However, there is some controversy about the validity of detecting metastasis by this approach. Since cancer cells spread to the red marrow space in the skeleton, FDG-PET imaging can effectively determine evidence of metastatic disease in the skeleton with high sensitivity and specificity. In contrast, skeletal imaging with radiotracers including $18 \mathrm{~F}-\mathrm{NaF}$ is of limited value because of poor sensitivity and specificity. We believe performing FDG-PET will obviate using either conventional or nonFDG-PET-based techniques for detection and monitoring of metastasis to the skeleton. Therefore, it is very timely for the scientific community to explore the role of $18 \mathrm{~F}-\mathrm{NaF}$ for assessing a variety of benign disorders and enhance the role of medical imaging numerous disorders of the skeletal system. In addition, $18 \mathrm{~F}-\mathrm{NaF}$ is a powerful tracer for detection of calcification in either the skeleton or soft tissues, including in the atherosclerotic plaques in the major and coronary arteries.

Since PET allows molecular imaging of underlying biological phenomena in many human disorders, its uses in medicine, including those related to disorders of the MSK system, are limitless. Research applications of PET-based molecular imaging provide a great opportunity for scientists to explore the enormous potential of this imaging modality in assessment of MSK disorders by utilizing various novel tracers that are being tested in the rest of the body. In these two issues, we have invited world MSK experts in PET/CT/MR imaging to provide the most up-to-date and latest information about their respective domains of expertise in MSK disorders. This comprehensive review allows clinicians and scientists to realize the vast potential of this new powerful approach, and in the long run, this will substantially benefit many patients with disabling diseases and disorders of this system. We hope these two issues of PET Clinics encourage future studies to collect the data needed to demonstrate the excellence of PET/CT/MR imaging in the evaluation of MSK disorders. The articles in these two issues illustrate what we know about the role of PET in the MSK system and where there are gaps and holes that should be filled, and also to help recognize opportunities and benefits.

The second issue of PET Clinics on MSK disorders provides a detailed overview of the basic principles and technical and methodological considerations of the performance of PET/CT/MR imaging for MSK applications in a practical manner and mainly focuses on benign pathologies. This will in particular help orthopedic surgeons and rheumatologists without deep knowledge of nuclear medicine and PET technology make a foundation for successive reading and have a better understanding of the technique and the acquisition protocols. Articles also cover the application of $\mathrm{PET} / \mathrm{CT} / \mathrm{MR}$ imaging with FDG and other radiopharmaceuticals in various clinical settings. These include applications of PET in primary benign soft tissue tumors of extremities and also osteoporosis, infection, and inflammation. There are 
several articles centered around the role of PET imaging of the MSK system in the context of competing imaging modalities, including spectral and dual-energy CT, dynamic contrast-enhanced MR imaging, contrast-enhanced CT imaging (including iodine overlay image), and also quantitative imaging.

Ali Gholamrezanezhad, MD Keck School of Medicine, University of Southern California (USC) 1520 San Pablo Street Los Angeles, CA 90033, USA

Ali Guermazi, MD, PhD Boston University School of Medicine Boston Medical Centre 820 Harrison Avenue, FGH Building, 3rd Floor Boston, MA 02118, USA
Ali Salavati, MD, MPH Department of Radiology University of Minnesota 420 Delaware Street Southeast Minneapolis, MN 55455, USA

Abass Alavi, MD, MD (Hon), $\mathrm{PhD}$ (Hon), DSc (Hon) Department of Radiology Hospital of the University of Pennsylvania 3400 Spruce Street Philadelphia, PA 19104, USA

E-mail addresses:

Ali.Gholamrezanezhad@UHhospitals.org

(A. Gholamrezanezhad)

Ali.Guermazi@bmc.org (A. Guermazi) salavati@gmail.com (A. Salavati) Abass.Alavi@uphs.upenn.edu (A. Alavi) 